\title{
FEATURE
}

\section{A cool spin on supercomputers}

To cite this article: Niladri Banerjee 2019 Phys. World 32 (4) 31

View the article online for updates and enhancements. 


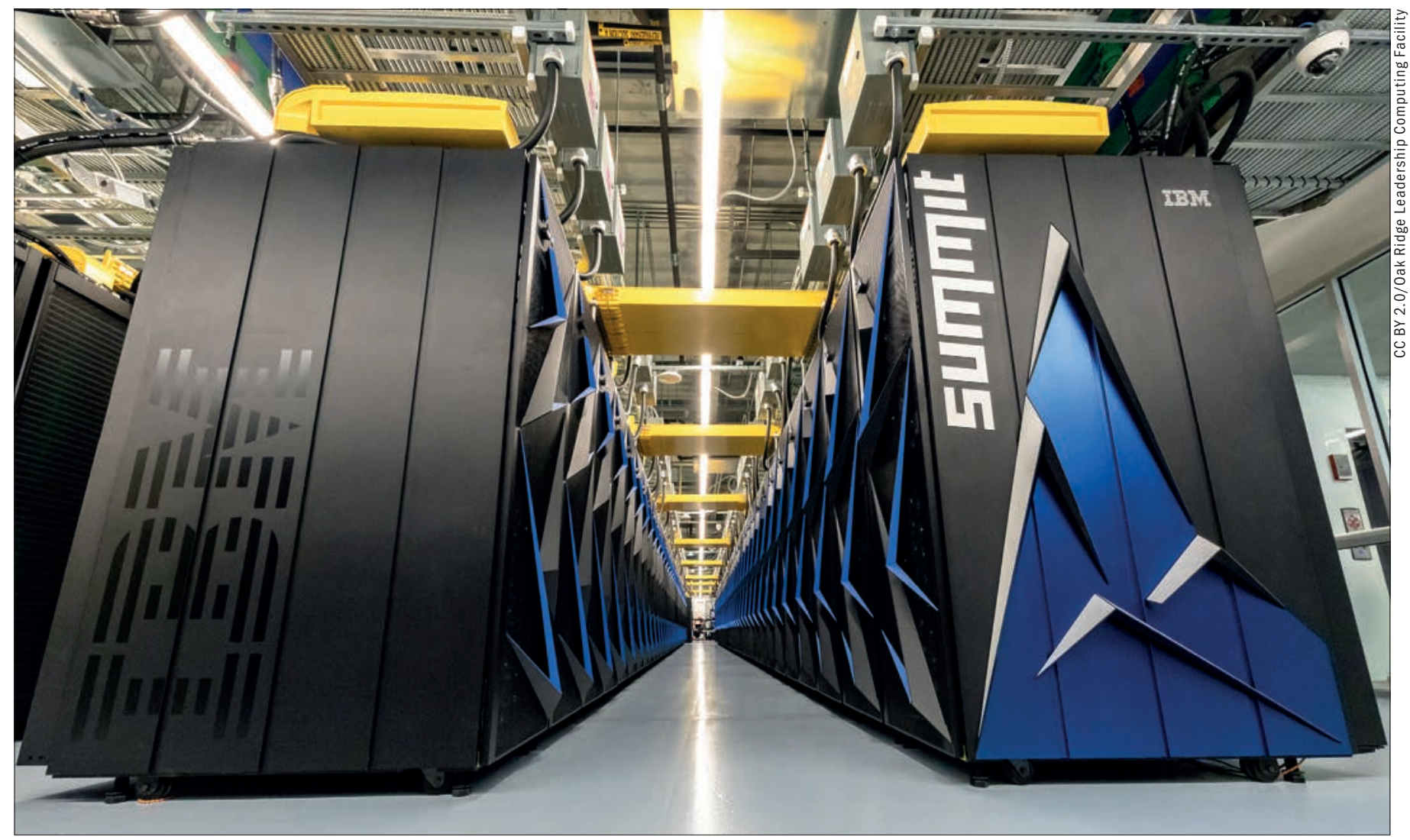

A cool spin on

supercomputers

Niladri Banerjee explains how the emerging field of "superconducting spintronics" could lead to a new generation of green supercomputers that use far less energy than previous devices

Summit. It's an apt name for the world's most powerful computer - a 200-petaflop behemoth at the Oak Ridge National Laboratory in Tennessee. Capable of performing $200 \times 10^{15}$ calculations per second, it's so big that its underground mainframe requires a room the size of two basketball courts. It's not cheap either, having cost an eye-watering $\$ 200 \mathrm{~m}$. Unveiled last year, Summit knocked the former champ - China's Sunway TaihuLight - off the supercomputer top spot as the US and China battle to outspend each other and create the planet's most formidable number cruncher.

Summit will be used for a myriad of "big-data" calculations such as advanced genomics and climate modelling. It's also the first supercomputer created specifically for artificial-intelligence (AI) applications including developing cancer treatments, designing advanced materials and understanding diseases such as Alzheimer's. But those benefits come at a price, which is that Summit requires as much power as a small town. And like all supercomputers, a huge fraction of that power is lost as heat.

That's the problem with supercomputers. Even with the latest cooling technology, which uses water to remove waste heat, it's tricky for engineers to keep the processor at the right operating temperature. It's hard to say exactly how much heat they generate, but Summit needs more than 17000 litres of water every minute to keep it running safely. And with plans for even faster, 1000-petaflop machines on the cards, the search is on for innovative ways to stop such devices guzzling so much energy.

\section{In a spin}

One solution could lie in the new field of "superconducting spintronics", which marries superconducting electronics with room-temperature spintronics. The first part of this union - superconducting electronics - relies on materials such as niobium in which current
Niladri Banerjee is a senior lecturer in physics at Loughborough University, UK, e-mail n.banerjee@ Iboro.ac.uk 


\section{Controlled spin}

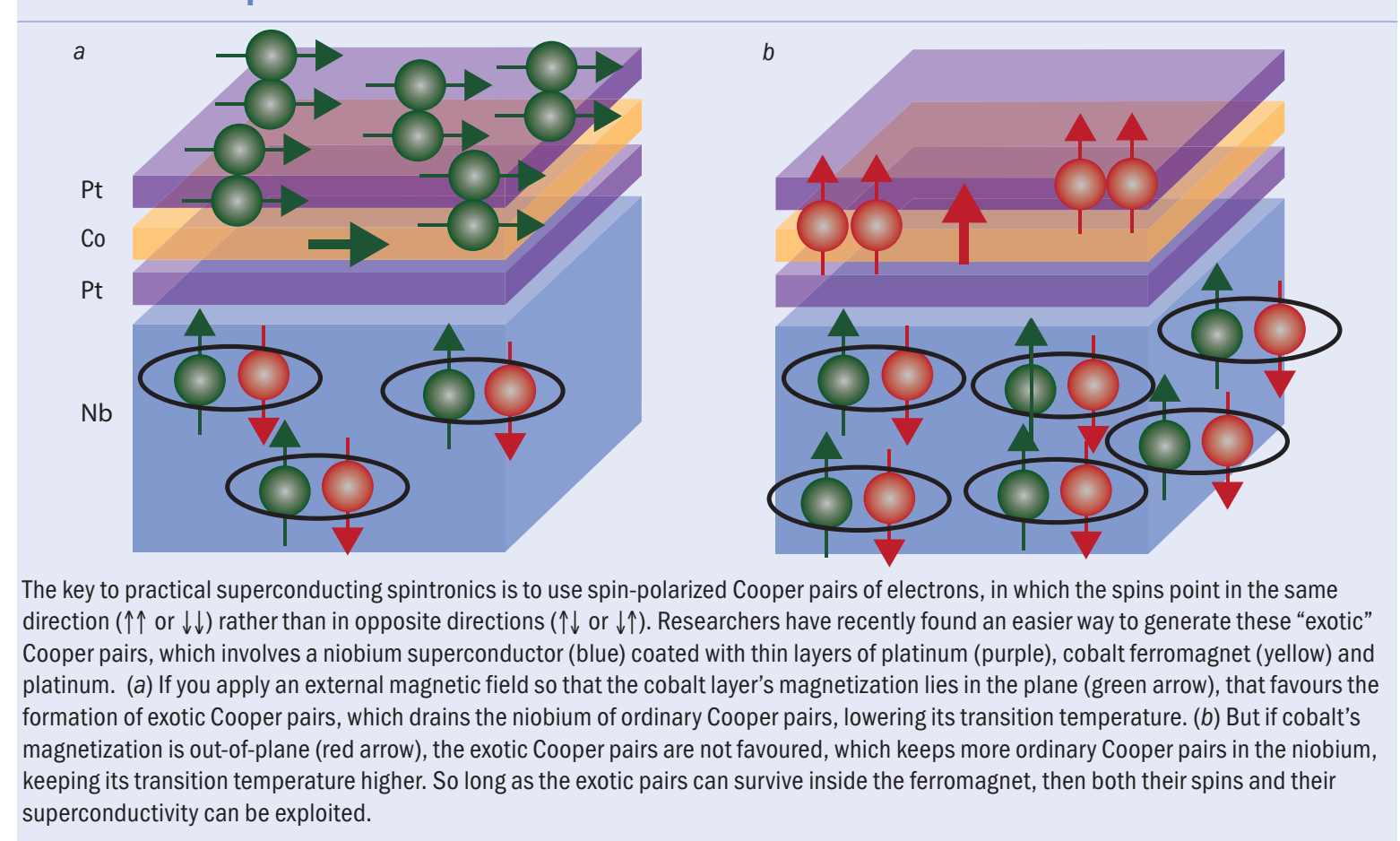

flows with no resistance and hence no heat loss. The overall energy loss, or "dissipation", from a supercomputer built entirely from such materials would be much reduced. Unfortunately, we don't yet know how to make the components of superconducting circuits as small as those found in conventional electronic circuits, roughly tens of nanometres in size.

The second half of the partnership - room-temperature spintronics - exploits the spin as well as the charge of electrons to store and process information. The spin - or intrinsic angular momentum - of an electron can point up $(\uparrow)$ or down $(\downarrow)$, which offers a way to efficiently store, process and manipulate information as $0 \mathrm{~s}$ and $1 \mathrm{~s}$. One way we can do this is using magnets a few atomic layers thick, made from cobalt, nickel or iron. The resulting current of spins lets us do interesting things like switch the magnetization using spin-polarized currents, in which all the spins point in the same direction.

Spintronic components aren't fiction. They already exist in computer hard drives as magnetic-field sensors consisting of copper sandwiched between two ultrathin ferromagnetic layers - one to spin-polarize the electrons injected into it and the other to analyse their orientation. Depending on the direction of an external magnetic field, the magnetic moments of the two layers either point in the same direction (a low spin-scattering, low-resistance state) or in opposite

By integrating superconductivity state). Known as giant magnetoresistance (GMR), the (no loss of heat) discovery of this effect led to Albert Fert and Peter with spintronic devices (lots of functionality), you get the best of both worlds electric current through these ultrathin magnetic layers, which leads to heat dissipation. For other similar spintronic applications such as switching a magnet using spin-polarized currents, this heat dissipation is far more. And this is where superconducting spintronics wins out. By integrating superconductivity (no loss of heat) with spintronic devices (lots of functionality), you get the best of both worlds.

\section{Exotic solution}

For superconducting spintronics to work, however, you cannot use conventional Cooper pairs, which are responsible for the remarkable ability of electrons to flow without losing any energy. Consisting of two electrons with spins pointing in opposite directions $(\uparrow \downarrow$ or $\downarrow \uparrow)$, their overall spin is zero, making them useless for spintronics. In 2001, however, three theoretical physicists - Sebastian Bergeret, Anatoly Volkov and Konstantin Efetov from the Ruhr University in Bochum, Germany - predicted the existence of "exotic" spin-polarized Cooper pairs, in which both spins point in the same direction $(\uparrow \uparrow$ or $\downarrow \downarrow)$. As long as these pairs can survive inside a ferromagnet, it ought to be possible to exploit both their spins and their superconductivity. Spin-polarized Cooper pairs offered the prospect of information-processing devices that are small, lose hardly any energy and have interesting functionalities.

It took physicists almost a decade, however, to firmly establish the existence of these Cooper pairs. The breakthrough came in a series of experiments carried out in 2010 by Mark Blamire and colleagues at the University of Cambridge and Norman Birge's group at Michigan State University (Science 32959 and Phys. Rev. Lett. 104 137002). The trouble is, it proved tricky to generate these spin-polarized Cooper pairs. They only form in artificial thin-film 
heterostructures of superconductors and two ferromagnets whose magnetic moments are perpendicular to each other.

Recently, however, researchers (including myself) at Loughborough University, Cambridge and the Norwegian University of Science and Technology have been able to hugely simplify the structure needed to generate spin-polarized Cooper pairs, opening the door to practical applications (Phys. Rev. $B 97$ 184521). Instead of having to delicately align several magnets to form the bridge between superconductivity and spintronics, we have found that similar effects are possible using a single magnet and the subtle relativistic effect of "spin-orbit coupling", which links the electron's spin with its motion around the nucleus of an atom.

What we did was to take a standard superconductor such as niobium and deposit on its surface an atomically thin layer of platinum, followed by a layer of ferromagnetic cobalt and finally another layer of platinum (figure 1). With the thicknesses of the platinum and cobalt layers carefully selected, we looked at the impact of tilting the magnetization of cobalt with respect to the film plane. When we applied an external magnetic field so that the magnetization of the cobalt layer was fully in the plane, we were surprised to find that the temperature at which the niobium starts superconducting fell dramatically compared with when there was no magnetic field. Although we knew that applying an external magnetic field reduces the superconducting transition temperature, the drop we saw was far bigger than expected.

Over the next two years, we performed rigorous experiments to nail down exactly why there is such a big fall in transition temperature. It turns out, it's all to do with the number of ordinary Cooper pairs. If the magnetism lies along the plane of the cobalt layer, the exotic Cooper pairs survive, which drains the superconductor of ordinary Cooper pairs and lowers its transition temperature. But if the magnetism of the cobalt layer points out of the plane of the film, the exotic Cooper pairs find it hard to survive, which means fewer Cooper pairs leak out, increasing its transition temperature.

The bottom line is that, thanks to spin-orbit coupling (in the platinum), we can control superconductivity (in the niobium) by adjusting the magnetization direction of a single magnet (the cobalt). The spin-orbit coupling lets us generate exotic Cooper pairs in a controllable way simply by adjusting the direction of a single ferromagnet. In essence, our stacked structure of platinum and cobalt has given us a spin-orbit-coupled ferromagnet. This effective communication between superconductivity and magnetism is like forming a bridge between the two phenomena, dramatically simplifying the structures needed to make useful circuit components in superconducting spintronics.

\section{Feeling green}

This form of superconducting spintronics, driven by spin-orbit coupling, makes it much easier to build the components needed for a fully functioning and practical superconducting spintronic circuit, taking us one step closer to more efficient supercomputers.

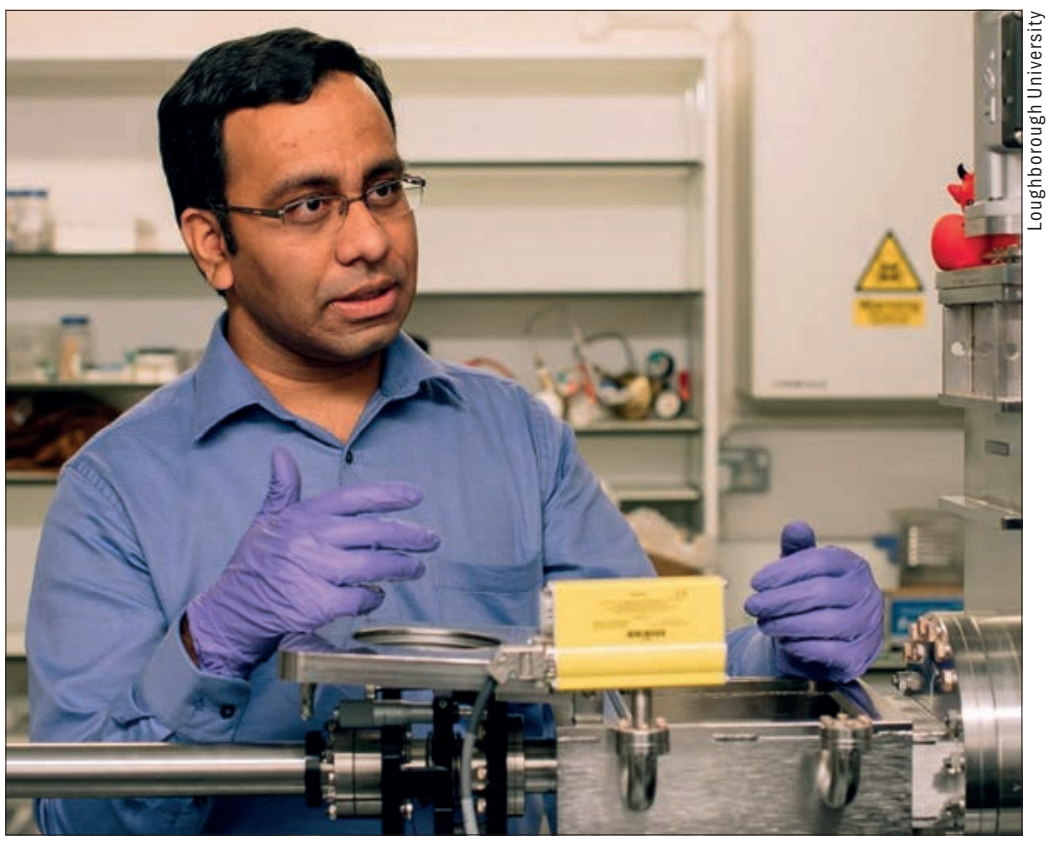

However, our work goes far beyond practical applications. It shows that three very exciting phenomena can coexist in this niobium-cobalt-platinum system: superconductivity, magnetism and spin-orbit coupling. Indeed, under specific conditions, entirely new phases of matter can emerge, including - in our case - a novel form of "magnetic superconductivity" (usually magnetism kills any superconducting behaviour). Such phases are not possible to generate in nature and exist only in these kinds of artificially engineered structures.

For those involved in superconducting spintronics, it is like being back in the mid-19th century when researchers had discovered electricity but had not yet invented the light bulb. What we now need is a device that can exploit our exotic Cooper pairs. One possibility would be to build a superconducting version of a "spin transfer torque" (STT) device - a conventional spintronics component in which the magnetization of a ferromagnet can be flipped using a spin-polarized current.

Such devices are already being used for STT magnetic random-access memory (STT-MRAM) chips. They are not only cheaper, use less energy and can store more information than conventional memory chips, but also let us precisely switch the magnet of one device without disrupting the magnetic alignment of nearby devices, which is always a danger when you switch a magnet using an external magnetic field.

In the spintronics version of STT, we could switch the magnetization of a nanomagnet by transferring spin angular momentum from the spin-polarized current. Building an STT device that uses superconducting spin-polarized currents might not be easy, but it would massively improve the energy efficiency of such devices. While it is hard to predict how information technology will evolve, I envisage today's supercomputers, which exploit only the charge of the electron, one day becoming obsolete - replaced by a new generation of superconducting spintronics supercomputers. Green supercomputers will then have finally arrived. 